\title{
Commentary
}

\section{The Art of Cost of Illness}

\author{
Anders Wimo* \\ KI Alzheimer's Disease Research Center, Department of Neurobiology, Care Sciences and Society, Karolinska \\ Institutet, Stockholm, Sweden
}

Accepted 22 September 2009

\section{INTRODUCTION}

The worldwide costs of dementia are US $\$ 315$ billion [1]. Monthly costs of Alzheimer's disease in Spain are $€ 1,426$ per month [2]. What is the purpose, value, and usefulness of such statements?

The number of Cost of Illness (CoI) studies is steadily increasing. For almost any high resource-consuming disease, there is some kind of CoI estimate available. There is also a kind of ongoing CoI rally. Like kids in the sandbox, advocates for one disease argue that "my disease is much more expensive than your disease", implicitly saying that a higher $\mathrm{CoI}$ demands more resources (than diseases with lower CoI).

Any health economist knows that this way of arguing is a misuse. But, so what; politicians and other decision makers in the allocation of resources are very sensitive to such policy-making statements. In a time when resources for care and research are scarce (which they of course always more or less will be!), perhaps not all, but many tricks seems to be defensible - "the end justifies the means"!

Behind any message there is an interest and a more or less hidden messenger - a drug company, a patient advocate organization/non-governmental organization (NGO), a research group looking for funding, etc.

Chastened economists also sarcastically say that if all CoI figures for various disorders are summarized, this

* Corresponding author: Anders Wimo, MD, PhD, KI Alzheimer's Disease Research Center, Department of Neurobiology, Care Sciences and Society, Karolinska Institutet, Stockholm, Sweden. E-mail: Anders.Wimo@ki.se. total figure will by far exceed the available resources. CoI studies also sometimes show disparate figures for a particular disorder for a country for the same period of time, making receivers of the information confused.

Some economists also do not appreciate CoI studies and argue that it is Cost Effectiveness studies that should be the focus for health economic research.

Is it really so bad? Well, not necessarily! However, we must be aware of the limitations of CoI studies. CoI studies per se cannot be used for cost effectiveness judgments or priority settings, since CoI studies have no intervention, no outcomes, and no comparators. There are also great differences in the comparisons between countries with different care systems.

However, CoI studies may be of great value when the economic impact of a disorder is under discussion, but the keyword is TRANSPARENCY. CoI studies can identify how resources and costs for a disorder are distributed among different "payers". Crucial here is the viewpoint, meaning that all relevant costs with associated payers should be included, that is a societal viewpoint (with opportunities to identify the relative impact on different payers). In dementia this implies above all that the unpaid care by informal carers should be given a monetary value. Quantification and costing informal care is a complicated issue [3]. In a Swedish CoI study [4], the ratio in the different cost estimates of informal care in the sensitivity analysis was a factor of 10 ! Thus, to make comparisons between different cost estimates of informal care, the included care activities (for example: is supervision/surveillance a care activity?), methods for quantification, and costing of 
informal care must be transparent. The importance and impact of informal care (or actually, the lack of it) is indeed highlighted by the viewpoint of NICE in UK, since informal care is not a part of their commission and accordingly not included in their judgments. One may argue that costs of informal care are not relevant since it is only the use of public spending or direct payments for other reasons that are of interest. However, let's assume that home services or the number of institutional resources are significantly reduced due to public economic problems. This cut down of measurable resources will probably be replaced by informal carers. If these informal carers are of working age, over to the long course of dementia disorders, there will be an opportunity cost for the forgone work that will not be produced. If informal care is not included in the calculations, a significant societal cost of dementia care will be missed.

CoI studies can have a "bottom-up" or "top-down" design (or a mix). With the top-down approach, the total national cost for a specific resource is distributed on different diseases, often by the use of registers. The bottom-up method starts from a defined sub-group (a local area or clinical samples, e.g., from memory clinics) with dementia, for example, and registers all costs of illness related to it, followed by an extrapolation to the total dementia population. The problem with topdown studies is that many resources and costs can not be identified and that the quality of the registers (e.g., diagnosis) can be questionable. The problem with the bottom-up method is the generalizability; how representative is a small area or a memory clinic to the whole dementia population?

Another situation where CoI studies may be of value is to make comparisons over time. What is happening when the prerequisites for care change over time? This can be made if the region and methods are similar. Crucial is the societal viewpoint, otherwise changes can appear as "cost reductions".

Even if CoI studies per se cannot be used for cost effectiveness judgments, CoI studies can be a support for Cost-Effectiveness studies. It is most often not possible to obtain the necessary underlying data for CostEffectiveness judgments from conventional randomized controlled trials (RCTs) even if such studies include empirical data of resource use, costs, and clinically relevant outcomes. For practical and perhaps ethical reasons, we cannot make disease covering RCTs for progressive, long-lasting, chronic disorders with a duration of ten years or more. Efficacy RCTs of, say 12-18 months, with piggy-backed health economic da- ta is one link for decision makers, but the relative short duration and the selected study populations highlight the need for other approaches such as economic modeling. Bottom-up CoI studies with detailed information of costs in relation to different states of a disease and with several background factors included are in that perspective of great value for further use in cost effectiveness studies/modeling (together with other sources of progression and intervention effects).

With all the reservations, methodological aspects and potential traps in mind, the Spanish study by Coduras in this issue [2] of the Journal of Alzheimer's Disease has its pros and cons. The study population, distributed along different severity states, is rather big and includes the most heavy cost drivers. It is, however, selective since the patients were recruited from neurology clinics, and severe concomitant diseases or absence of a reliable caregiver excluded patients. It has a longitudinal approach and the principles for assessing caregiver time are clearly stated and a variation of the unit costs for informal care was included in the sensitivity analysis. The study also confirms that functional capacity is a better predictor of costs than cognition, but it would have been better to have a specific behavioral scale than the subscale in the Blessed Dementia Rating Scale.

The main findings, that the costs of informal care is the major cost driver and that the costs of institutional long-term care is low in Spain, is not a surprise and has been found in studies from other Mediterranean countries in contrast to Northern Europe where the cost of institutional care is the major cost driver [5,6]. The results indeed confirm the need of a societal viewpoint including costs of informal care.

The potential influence of policy makers, advocate organizations/NGOs, and drug companies (as in this study with Pfizer as sponsor) is not necessarily a problem either. As long as the methods used are accurate and transparent and the role of the sponsors is clearly defined, it is, as usual, up to the readers to use and critically interpret the information in a CoI study.

\section{DISCLOSURE STATEMENT}

Author's disclosure available online (http://www.jalz.com/disclosures/view.php?id=165).

\section{REFERENCES}

[1] Wimo A, Jönsson L, Winblad B (2007) An estimate of the total worldwide societal costs of dementia in 2005. Alzheimers Dement 3, 81-91. 
[2] Coduras A, Rabasa I, Frank A, Bermejo-Pareja F, Lopez-Pousa S, Lopez-Arrieta JM, Del Llano J, Leon T, Rejas J (2010) Prospective one-year cost-of-illness study in a cohort of patients with dementia of Alzheimer's disease type in Spain: The ECO study. J Alzheimers Dis 19(2) 587-601.

[3] van den Berg B, Brouwer WB, Koopmanschap MA (2004) Economic valuation of informal care. An overview of methods and applications. Eur J Health Econ 5, 36-45.

[4] Wimo A, Johansson L, Jonsson L (2009) [Prevalence study of societal costs for dementia 2000-2005. More demented people-but somewhat reduced costs per person]. Lakartidningen 106, 1277-1282.

[5] Jonsson L, Wimo A (2009) The cost of dementia in europe: a review of the evidence, and methodological considerations. Pharmacoeconomics 27, 391-403.

[6] Wimo A, Jönsson L, Gustavsson A (2008) The cost of illness and burden of dementia in Europe. In Dementia in Europe Yearbook 2008, Alzheimer Europe, Luxembourg, pp. 67-71. 